\title{
Development, research and metrological analysis of the measuring channel of a fiber- optic sensor for fluid flow parameters used in information-measuring systems
}

\author{
Elena Badeeva $^{1}$, Elena Shachneva ${ }^{1, *}$, Alexandr Udalov $^{1}$, and Tatiana Murashkina ${ }^{1}$ \\ ${ }^{1}$ FSBEI HE Penza State University, Chair Instrument making, 440026 Penza, Krasnaya, 40, Russia
}

\begin{abstract}
The article proposes a device for measuring parameters of liquid flows (volume and velocity) (FOSLFP). A physico-mathematical model of optical signal conversion in a fiber-optic flux transducer of the proposed sensor is developed, in which the perceiving element is a truncated cylinder mounted on the inner surface of the bellows, experiencing angular bending during measurements. The conversion of optical signals is carried out using a two-way mirror plate mounted on the outer surface of the bellows in an open fiber optical channel. A circuitanalytical model of a new variant of a differential circuit for converting optical signals to a FOSLFP was developed, in accordance with which a metrological model of the sensor was developed to determine the possible sources of measurement errors. The nominal and real functions of the FOSLFP transformation were derived, the additive and multiplicative components of the errors of the two measuring channels of the sensor and the ways to reduce them were determined.
\end{abstract}

\section{Introduction}

The safety of operation of technical systems and objects created in high-tech branches of science and technology, to which the decree of the President of the Russian Federation dated 07.07.2011 referred as rocket and space and aviation industries, and nuclear power and many other industries, today largely depends on the accuracy of measurements of fluid flows [1].

The problem of creating and improving methods and means of measuring the parameters of substances with specific properties (aggressiveness, unsteadiness of physical and chemical characteristics, high viscosity) and functioning in various difficult operating conditions, despite some progress, remains very relevant [2].

\section{Materials and Methods}

The authors of this article propose to use a new, safe, highly reliable fiber-optic sensor of liquid flow parameters (FOSLFP). The principle of operation FOSLFP is as follows (figure $1)$.

\footnotetext{
* Corresponding author: e_shachneva@mail.ru
} 


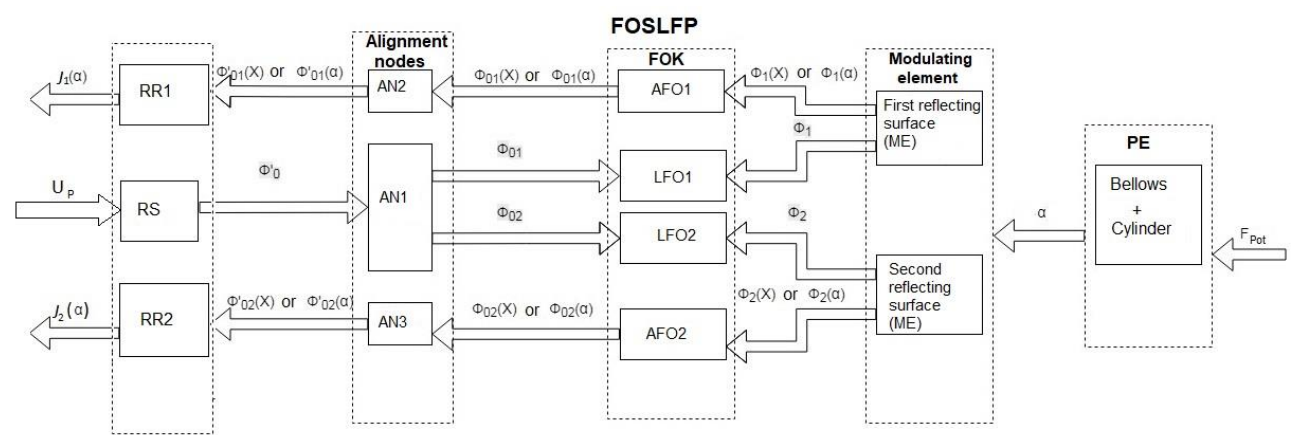

Fig.1. Structural and analytical scheme of FOSLFP parameters of liquid flows

The electrical signal $U_{P}$, input to the input of the radiation source of the $R S$, is converted by means of an electro-optical converter of the RS into an optical signal $\Phi$, part of which $\Phi{ }^{\prime}{ }_{0}$ is fed to the input of the fiber optic cable of the FOC through the alignment unit of the AN1. According to the supply optical fiber of the LFO, the light flux is transferred to the measuring zone of the fiber optic converter FOC, where its intensity $\Phi_{0}$ changes under the action of the measured physical quantity $\alpha$. The part of the light flux $\Phi_{\mathrm{ME}}(\alpha)$, modulated by the modulating element $\mathrm{ME}$ in the function of the measured physical quantity $\alpha$ of the flux force F, enters the diverter optical fiber AFO, is transmitted through them through the AN2 to the radiation receiver RR, where the photoelectric conversion takes place. The electrical signal I $(\alpha)$ is removed from the output optoelectronic unit [3].

Under the influence of the $\mathrm{F}_{\text {Pot }}$ force produced by the liquid flow, the SE element will deviate by some angle $\alpha$, and the reflecting plate of the RP will deviate by the same angle.

The light fluxes $\Phi_{01}$ and $\Phi_{02}$ from the RS according to the LFO1 and LFO2 are sent to the measuring zone to the first and second reflecting surfaces of the plate, respectively. As the angle $\alpha$ is varied, the intensities of the reflected light fluxes $\Phi_{1}(\alpha)$ and $\Phi_{2}(\alpha)$, respectively, change [4-6].

To determine the possible sources of measurement errors and to find ways to reduce them, a metrological analysis of the FOSLFP channel was carried out. For this, a metrological structural scheme of the sensor was developed [7-8].

As shown above, in the differential FOSLFP, the measured flow strength $\mathrm{F}$ is converted into an reflecting plate RP offset at an angle $\alpha$, so the schematic-analytical model of the new version of the differential transformation scheme will have the form shown in Figure 2.

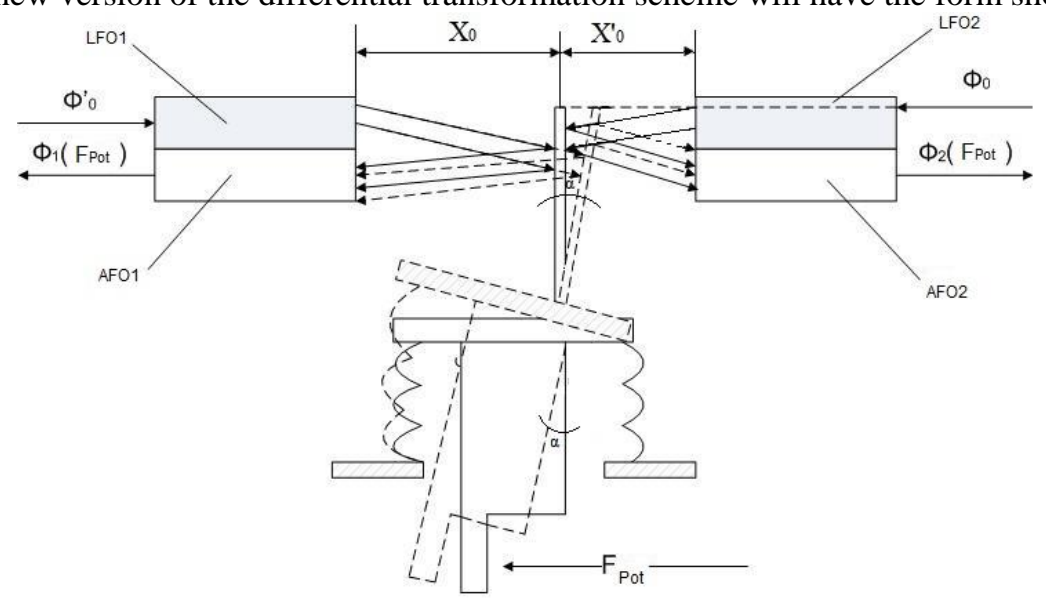

Fig. 2. - Diagram of a fiber-optic converter of the force of a fluid flow with RP 


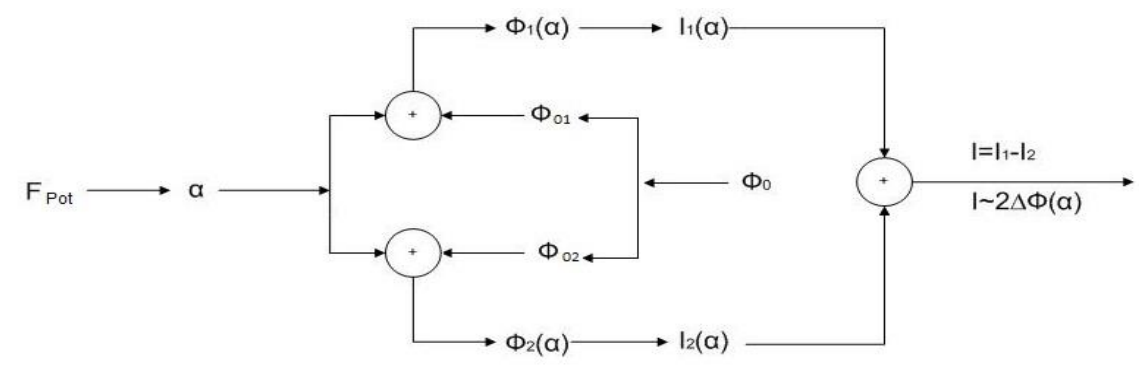

Fig. 3. - Schematic-analytical model of a new version of the differential signal conversion scheme for optical signals FOSLFP

Based on the above, as an example, the constructive, structural and functional diagrams of the FOSLFP are presented, shown in Figure 4, to explain the process of reducing errors in differential signal conversion in FOSLFP, where:

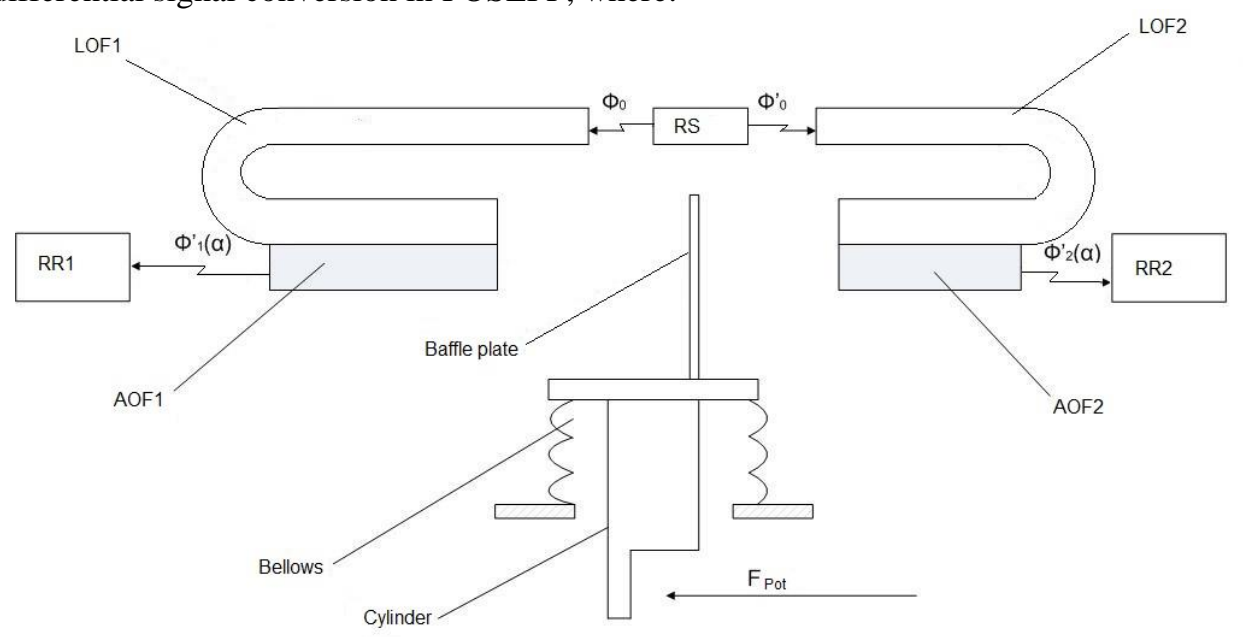

a)

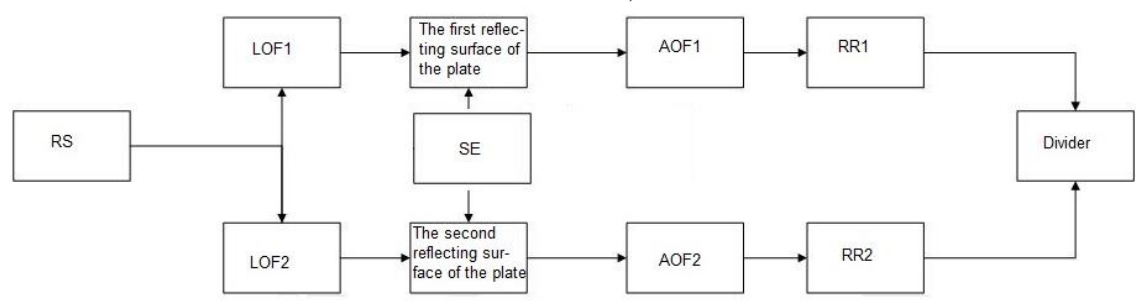

b)

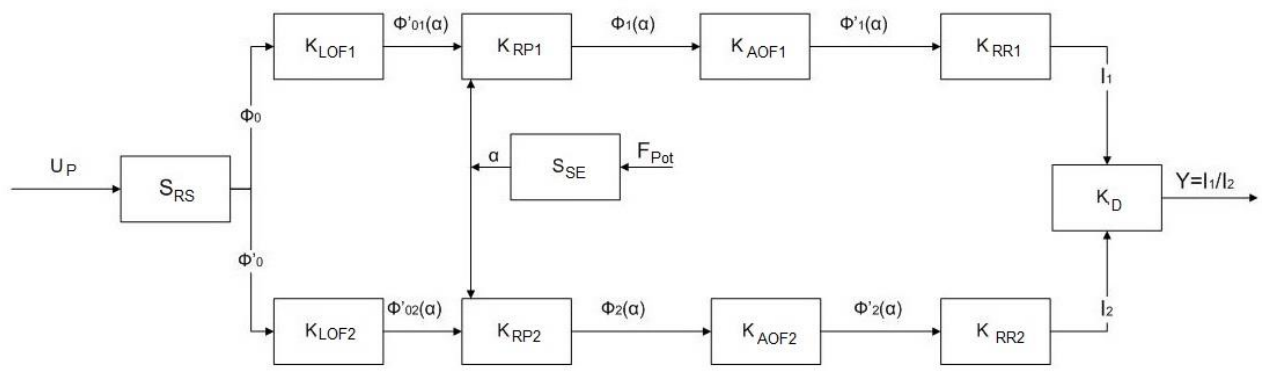

c) 
RS - source of radiation; LFO1, LFO2 - lead-in optical fiber 1 and 2; AFO1, AFO2 - the allotment optical fiber 1 and 2; RR1, RR2 - radiation receivers of the first and second

Fig.4. - Differential FOSLFP measuring channel

a - constructive scheme; $b$ - structural diagram; $\mathrm{c}$ - functional diagram

$S_{S E}$ - sensitivity of SE;

$K_{R P 1}, K_{R P 1}$ - coefficients of transformation of the first and second surfaces of the RP, respectively;

$S_{R S}$ - sensitivity of the RS;

$K_{L F O 1}, K_{L F O 2}$ - the transmission coefficient of the optical channel RP - LFO1 and RP LFO2 respectively;

$K_{A F O 1}, K_{A F O 2}$ - transmission coefficient of the first and second optical channels RP AFO1, respectively;

$K_{R R I}, K_{R R 2}$ - the sensitivity of the first and second RR;

$K_{D^{-}}$coefficient of the divisor transformation.

Mathematically, the conversion function is written:

$$
Y=P_{\mathrm{RS}} S_{\mathrm{SE}}\left(K_{\mathrm{LFO} 1} K_{\mathrm{RP} 1} K_{\mathrm{AFO} 1} S_{\mathrm{RR} 1} / K_{\mathrm{LFO} 2} K_{\mathrm{RP} 2} K_{\mathrm{AFO} 2} S_{\mathrm{RR} 2}\right) K_{\mathrm{D}} F
$$

where $Y$ - output value (for example, voltage);

$F$ - measured fluid flow strength.

In the absence of a flow force at $F=0, \Phi_{1}(F)=\Phi_{2}(F)$. If the RP is shifted, then the light fluxes are redistributed between the receiving fibers: the optical signal $\Phi_{1}-\Delta \Phi$ is fed into the first ALF, and the optical signal $\Phi 2+\Phi$ is fed into the second ALF. If these signals are fed to the RR, and then to the subtractor, a signal proportional to the difference in the radiation fluxes is formed:

$$
I \sim\left(\Phi_{1}-\Delta \Phi\right)-\left(\Phi_{2}+\Delta \Phi\right)=2 \Delta \Phi,
$$

That network is a doubling of the sensitivity of the conversion.

If we form the ratio of the difference of the signals to their sum, then:

$$
\left[I_{1}(F)-I_{2}(F)\right] /\left[I_{1}(F)+I_{2}(F)\right] \sim\left(\Phi_{1}-\Phi_{2}\right) /\left(\Phi_{1}+\Phi_{2}\right) .
$$

Consider the structural metrological model of the sensor (Figure 5). 


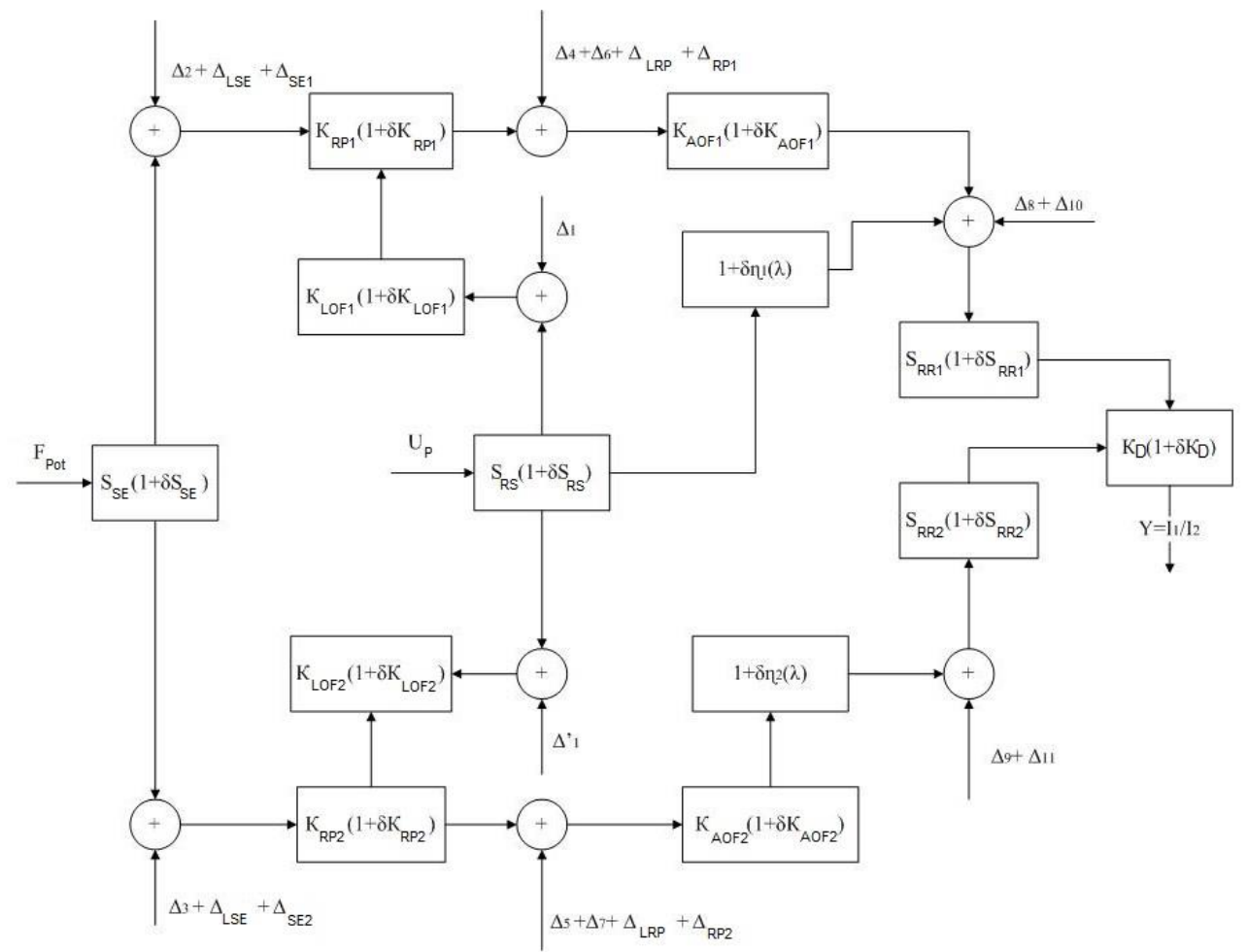

Fig.5. - Structural metrological model of FOSLFP

In figure 5 the following designations are accepted:

$\Delta_{1}$ - the error of alignment of LFO1 relative to RS;

$\Delta_{1}^{\prime}$ - the error of alignment of LFO1 relative to RS;

$\Delta_{2}, \Delta_{3}, \Delta_{4}, \Delta_{5}$ - inaccuracy due to the inaccuracy of the initial installation of the SE, with respect to the $\mathrm{LFO}$ and $\mathrm{AFO}$;

$\Delta_{6}, \Delta_{7}$ - inaccuracies due to inaccuracies in aligning the LFO and AFO with respect to each other;

$\Delta_{S E 1}, \Delta_{S E 2}$ - inaccuracies due to inaccuracy in the manufacture of SE introduced into the first and second channels;

$\Delta_{R P 1}, \Delta_{R P 2}$ - inaccuracies due to inaccuracy in the manufacture of RP introduced into the first and second channels;

$\Delta_{8}, \Delta_{9}$ - the errors in the alignment of AFO1 and AFO2 relative to RR1 and RR2;

$\Delta_{10}, \Delta_{11}$ - errors in the spectral matching of RS and RR1, and RR2;

$\Delta_{L R P 1}, \Delta_{L R P 2}$ - errors in the linearity of the optical signal transformation functions in the first and second measurement channels of the IR, caused by the reflecting plate;

$\Delta_{L S E 1}, \Delta_{L S E 2}$ - errors in the linearity of the transformation functions of the SE of the first and second channels;

$K_{L F O 1}, K_{L F O 2}, \delta K_{A F O 1}, \delta K_{A F O 2}$ - errors due to changes in the light transmittance of LFO and AFO, respectively, in the bending of the FOC; 
$\delta S_{S E}$ - the sensitivity of the sensitivity of the SE, due to changes in its parameters with a change in ambient temperature, mechanical effects, etc .;

$\delta K_{R P 1}, \delta K_{R P 2}$ - errors introduced into the first and second channels, respectively, from the displacements of the RP under the influence of perturbing forces on it and changes in the longitudinal and transverse dimensions under the action of temperature;

$\delta S_{R S}$ - the error caused by the change in power and the radiation pattern of the RS with a change in ambient temperature, supply voltage, etc .;

$\delta S_{R S 1}, \delta S_{R S 2}$ - the error from the change in the integrated current sensitivity RS1 and RS2 with a change in the temperature of the surrounding environment, the supply voltage;

$\delta \eta_{1}(\lambda), \delta \eta_{2}(\lambda)$ - the error of spectral matching of RS and RR1, and RR2, respectively, with a change in temperature;

$\delta K_{D}$ - the error in the conversion factor of the dividing device when the ambient temperature and the supply voltage change.

Nominal conversion functions:

1) For the first measuring channel MC:

$$
I_{N I}=S_{S E} P_{R S} K_{R P I} K_{L F O I} K_{A F O I} S_{R R} F ;
$$

2) For the second MC:

$$
I_{N 1}=S_{S E} P_{R S} K_{R P 2} K_{L F O 2} K_{A F O 2} S_{R R} F .
$$

Absolute errors in the transformation of each of the infrared are determined as follows:

$$
\Delta I_{1}=I_{R 1}-I_{N 1}, \quad \Delta I_{2}=I_{R 2}-I_{N 2},
$$

where $I_{R 1}, I_{R 2}$ - real conversion function.

The multiplicative component of the error is:

1) For the first MC

$$
\delta J_{1}=\sqrt{\delta P_{\mathrm{RS}}^{2}+\delta K_{\mathrm{LFO} 1}^{2}+\delta S_{\mathrm{SE}}^{2}+\delta K_{\mathrm{RP} 1}^{2}+\delta K_{\mathrm{AFO} 1}^{2}+\delta^{2} \eta_{1}(\lambda)+\delta S_{\mathrm{RR} 1}^{2}},
$$

2) For the second IR

$$
\delta J_{2}=\sqrt{\delta P_{\mathrm{RS}}^{2}+\delta K_{\mathrm{LFO} 2}^{2}+\delta K_{\mathrm{SE}}^{2}+\delta K_{\mathrm{RP} 2}^{2}+\delta K_{\mathrm{AFO} 2}^{2}+\delta^{2} \eta_{2}(\lambda)+\delta S_{\mathrm{RR} 2}^{2}} .
$$

Additive component of error:

1) For the first MC

$$
\begin{gathered}
\Delta J_{1}=K_{\mathrm{LFO} 1} \Delta_{1}+K_{\mathrm{RP} 1}\left(\Delta_{\mathrm{LSE} 1}+\Delta_{2}+\Delta_{\mathrm{SE} 1}\right)+ \\
K_{\mathrm{AFO} 1}\left(\Delta_{4}+\Delta_{6}+\Delta_{\mathrm{LRP} 1}+\Delta_{\mathrm{RP} 1}\right)+S_{\mathrm{RR} 1}\left(\Delta_{8}+\Delta_{10}\right),
\end{gathered}
$$

2) For the second $\mathrm{MC}$

$$
\begin{gathered}
\Delta J_{2}=K_{\mathrm{LFO} 2} \Delta_{1}+K_{\mathrm{RP} 2}\left(\Delta_{\mathrm{LSE} 2}+\Delta_{3}+\Delta_{\mathrm{SE} 3}\right)+ \\
K_{\mathrm{AFO} 2}\left(\Delta_{5}+\Delta_{7}+\Delta_{\mathrm{LRP} 2}+\Delta_{\mathrm{RP} 2}\right)+S_{\mathrm{RR} 2}\left(\Delta_{9}+\Delta_{12}\right) .
\end{gathered}
$$

Non-linear component:

1) For the first MC

2) For the second $M C$

$$
\Delta J_{L 1}=K_{R P 1}\left(\Delta_{\mathrm{LSE} 1}+\Delta_{\mathrm{SE} 1}\right)+K_{\mathrm{AFO} 1} \Delta_{\mathrm{LRP} 1},
$$

$$
\Delta J_{L 2}=K_{R P 2}\left(\Delta_{\mathrm{RP} 2}+\Delta_{\mathrm{SE} 2}\right)+K_{\mathrm{AFO} 2} \Delta_{\mathrm{LRP} 2} .
$$


The errors $\Delta 8, \Delta 9$ of the position of the ALF relative to the radiation receivers are practically equal to zero. Errors $\Delta 10$ and $\Delta 11$ of the spectral matching of RS and RR will be negligible if the RS - RR pairs are chosen correctly $[9,10]$.

The multiplicative errors $\delta \mathrm{K}_{\mathrm{LFO} 1}, \delta \mathrm{K}_{\mathrm{LFO} 2}, \delta \mathrm{K}_{\mathrm{AFO} 1}, \delta \mathrm{K}_{\mathrm{AFO} 2}$, caused by the change in the light transmittance of LFO and AFO under the influence of radiation, mechanical factors (impacts, vibration, linear acceleration), caused by cable bends, etc [11-13].

Technological errors due to inaccuracy in the manufacture of RP $\Delta_{\mathrm{RP} 1}$ and $\Delta_{\mathrm{RP} 2}$ are comparable with the above-mentioned errors in significance. To reduce the error data up to permissible values, it is possible to constructively use the advanced manufacturing technology of FOSLFP [14-16].

The errors in the conversion of each of the channels without taking into account errors that can be neglected will be determined by the expressions:

$$
\begin{aligned}
& \Delta I_{1}=K_{\mathrm{RP} 1}\left(\Delta_{\mathrm{LSE} 1}+\Delta_{2}+\Delta_{\mathrm{SE} 1}\right)+K_{\mathrm{AFO} 1}\left(\Delta_{4}+\Delta_{6}+\Delta_{\mathrm{LRP} 1}+\Delta_{\mathrm{RP} 1}\right)+\delta S_{\mathrm{SE}} F, \\
& \Delta I_{2}=K_{\mathrm{RP} 2}\left(\Delta_{\mathrm{LSE} 2}+\Delta_{3}+\Delta_{\mathrm{SE} 2}\right)+K_{\mathrm{AFO} 2}\left(\Delta_{5}+\Delta_{7}+\Delta_{\mathrm{LRP} 2}+\Delta_{\mathrm{RP} 2}\right)+\delta S_{\mathrm{SE}} F,
\end{aligned}
$$

and the real conversion function is written:

$$
\begin{gathered}
Y_{\mathrm{R}}=S_{\mathrm{SE}}\left(1+\delta S_{\mathrm{SE}}\right) S_{\mathrm{RS}} K_{\mathrm{LFO} 1} K_{\mathrm{D}}\left(1+\delta K_{\mathrm{D}}\right) U_{\mathrm{P}} F \times \\
\times\left[\left(K_{\mathrm{RP} 1}+\Delta_{\mathrm{LRP} 1}+\Delta_{2}+\Delta_{\mathrm{RP} 1}\right) S_{\mathrm{SE} 1}\left(K_{\mathrm{AFO} 1}+\Delta_{4}+\Delta_{6}+\Delta_{\mathrm{LRP} 1}+\Delta_{\mathrm{RP} 1}\right) /\right. \\
\left./\left(K_{\mathrm{RP} 2}+\Delta_{\mathrm{LSE} 2}+\Delta_{3}+\Delta_{\mathrm{SE} 3}\right) S_{\mathrm{RR} 2}\left(K_{\mathrm{AOF} 2}+\Delta_{5}+\Delta_{7}+\Delta_{\mathrm{LRP} 2}+\Delta_{\mathrm{RP} 2}\right)\right] .
\end{gathered}
$$

It is obvious that in the differential scheme $\Delta_{\mathrm{LSE} 1} \approx \Delta_{\mathrm{LSE} 2}, \Delta_{2} \approx \Delta_{3}, \Delta_{\mathrm{SE} 1} \approx \Delta_{\mathrm{SE} 2}, \Delta_{4} \approx \Delta_{5}, \Delta_{6} \approx \Delta_{7}$, $\Delta_{\text {LRP1 }} \approx \Delta_{\text {LRP2}}$, then

$$
\begin{aligned}
& Y_{\mathrm{R}}=S_{\mathrm{SE}}\left(1+\delta S_{\mathrm{SE}}\right) S_{\mathrm{RS}} K_{\mathrm{LFO} 1}\left[K_{\mathrm{RP} 1} S_{\mathrm{RR} 1}\left(K_{\mathrm{AFO} 1}+\Delta_{\mathrm{RP} 1}\right) /\right. \\
& \left./ K_{\mathrm{RP} 2} S_{\mathrm{RR} 2} K_{\mathrm{LFO} 2}\left(K_{\mathrm{AFO} 2}+\Delta_{\mathrm{RP} 2}\right)\right] K_{\mathrm{VU}}\left(1+\delta K_{\mathrm{VU}}\right) U_{\mathrm{P}} F .
\end{aligned}
$$

According to the literature, the error of the $\mathrm{K}_{\mathrm{VU}}$ is about $0.25 \%$ [17].

The technological errors $\Delta_{\mathrm{RP} 1}, \Delta_{\mathrm{RP} 2}$, caused by inaccuracy in the manufacture of RS, can be excluded or reduced by constructive and technological means $[18,19]$.

Differential transformation of signals in FOSLFP allows you to reduce most of its errors [20].

\section{Results}

On the example of the metrological analysis of FOSLFP it was shown that the differential transformation of optical signals with identical manufacturing of two measuring channels allows to improve the metrological characteristics of FOSLFP 2-3 times.

The metrological analysis of the channel for measuring the flow of liquid in the water supply and sewerage system made it possible to conclude that the use of a fiber-optic sensor for the parameters of liquid flows improves the metrological characteristics of the system.

\section{References}

1. I.E. Idelchik Reference book on hydraulic resistance (1975)

2 T.I. Murashkina, A.V. Arkhipov, A.G. Pivkin, D.I. Proceedings of the International Symposium Reliability and quality, 15 (2) (2012)

3. O.V. Yurova Technological procedures for manufacturing fiber-optic converters of linear and angular displacements of the reflective type: the abstract of the dis. ... Cand.Tech.Sci .: 05.11.14 ( 2011)

4. T.I. Murashkina, A.Yu. Udalov, E.A. Shachneva problems of science Materials of the International Scientific and Practical Conference. 272-274 (2016) 
5. E.A. Badeeva, L.N. Kolomiets, N.P. Krivulin Development of the theory of the distribution of the light flux in the optical system of the physical density of physical quantities of the reflective type: Monograph (2008)

6. Murashkina T.I., Badeeva E.A., Shachneva E.A., Istomina T.V., Udalov A.Yu., Serebryakov D.I. Biomedical Engineering, 295-299 (5) (2016)

7. E.A. Shachneva, T.I. Murashkina Journal of Physics, (735) 012-036 (2016)

8. Murashkina T.I., Badeeva E.A., Yurova O.V. Biotechnosphere, (43) 21-25 ( 2016)

9. E.A. Badeeva Theoretical bases of designing amplitude optical fiber pressure sensors with an open optical channel: Monograph (2004)

10. T.V. Alekseeva, N.S. Galdin, E.B. Sherman Hydraulic machines and hydraulic drive of mobile machines (1994)

11. T.M. Bashta Mechanical engineering hydraulics (1971)

12. V.A. Vasilchenko Hydraulic equipment of mobile machines (1983)

13. V.B. Weinberg Optics of light guides (1977)

14. A.M. Polyansky, V.A. Polyansky, A.N. Pronin, V.N. Gorobey, A.A. Chernyshenko. Bulletin of the Metrologist (4) 30-33 (2012)

15. E.A. Badeeva, M.M. Savochkina Innovative, information and communication technologies. (1) (245-250) 2017

16. A.D. Girgidov. E.N.Kozhevnikova, A.I.Laksberg, E.A. Loktionova. Mechanics of fluid and gas (hydraulics) (2007)

17. A.Zh. Muftakhov Hydrodynamic foundations of the forecast of flooding of industrial sites and filtration calculations of protective drainage in complex hydrogeological conditions. The dissertation author's abstract on competition of a scientific degree of the doctor of engineering science (1975)

18. T.I. Murashkina, T.V. Istomina, E.A. Badeeva New information technologies in medicine, biology, pharmacology and ecology materials 183-190 (2015)

19. A.Yu. Udalov, E.A. Shachneva, O.V. Yurova Problems of automation and control in technical systems Collection of articles of the International scientific and technical conference dedicated to the 70th anniversary of the Victory in the Great Patriotic, 260265 (2015)

20. EA. Shachneva, T.I. Murashkina, A.Yu. Udalov Actual problems of medical science and education 322-325 (2015) 